\title{
On the relation between gene flow theory and genetic gain
}

\author{
Piter Bijma ${ }^{\mathrm{a} *}$, John A. Woolliams ${ }^{\mathrm{b}}$ \\ a Animal Breeding and Genetics Group, Wageningen Institute of Animal Sciences, \\ Wageningen University, P.O. Box 338, Wageningen, The Netherlands \\ b Roslin Institute (Edinburgh), Roslin, Midlothian EH25, 9PS, UK
}

(Received 29 June 1999; accepted 19 October 1999)

\begin{abstract}
In conventional gene flow theory the rate of genetic gain is calculated as the summed products of genetic selection differential and asymptotic proportion of genes deriving from sex-age groups. Recent studies have shown that asymptotic proportions of genes predicted from conventional gene flow theory may deviate considerably from true proportions. However, the rate of genetic gain predicted from conventional gene flow theory was accurate. The current note shows that the connection between asymptotic proportions of genes and rate of genetic gain that is embodied in conventional gene flow theory is invalid, even though genetic gain may be predicted correctly from it.
\end{abstract}

genetic gain / gene flow / overlapping generations / selection response

Résumé - Note sur la relation entre le calcul de flux des gènes et le progrès génétique. Dans la méthode classique de calcul de la transmission des gènes, le taux de progrès génétique est calculé comme la somme des produits de la différentielle de sélection génétique et de la proportion asymptotique des gènes provenant des groupes âge-sexe. Des études récentes ont montré que les proportions asymptotiques de gènes prédites à partir de la méthode classique de calcul des flux de gènes peuvent dévier considérablement des proportions réelles. Par contre, le progrès génétique est prédit à partir de cette même méthode avec une bonne précision. La présente note montre que le lien entre le flux des gènes et le progrès génétique, tel qu'il apparaît dans la méthode classique de calcul des flux des gènes n'est donc pas correct, même si le progrès génétique peut être correctement prédit à partir de ladite méthode.

progrès génétique / flux de gènes / génération chevauchante / réponse à la sélection

\section{INTRODUCTION}

In conventional gene flow theory developed by Hill [2], the rate of genetic gain is calculated as the summed products of genetic selection differential and

\footnotetext{
* Correspondence and reprints

E-mail: Piter.Bijma@alg.vf.wau.nl
} 
asymptotic proportion of genes deriving from sex-age groups. Recent studies $[1,6]$ have shown that asymptotic proportions of genes predicted from conventional gene flow theory may deviate considerably from true proportions. However, rate of genetic gain predicted from conventional gene flow theory was accurate. The aim of the current note is to clarify this apparent contradiction.

\section{CONVENTIONAL GENE FLOW THEORY}

Conventional gene flow theory developed by Hill [2] is a method for predicting responses and discounted returns from selection in populations with overlapping generations. In conventional gene flow theory, asymptotic response from a single cycle of selection is calculated as the sum of the product of the asymptotic proportion of genes deriving from the different age-sex classes and their genetic selection differential (Eq. (12) of [2]). Since the result agrees with the ratio of mean genetic selection differential $(\bar{S})$ to mean generation interval (L) [5], Hill [2] concluded that the product of asymptotic proportion of genes and selection differential is equal to the rate of genetic gain.

Hill [2] presented two alternative formulations which are equivalent. First, asymptotic proportions of genes of sex-age groups were defined as the sum of proportions due to current and subsequent matings, and selection differentials were expressed relative to the previous mating. Second, asymptotic proportions of genes were defined as the proportion due to the current mating only, and selection differentials were expressed as a deviation of the mean of the whole contemporary sex-age group (Hill refers to this as "cumulative selection differential"). Here we will use the second formulation, so that genetic gain from conventional gene flow theory equals ("alternative formulation of (12)", p. 125 in $[2])$ :

$$
\Delta G=\sum_{k} r_{k, \infty} S_{k}
$$

where $r_{k, \infty}$ is the asymptotic proportion of genes deriving from the $k$ th sexage group, $S_{k}$ is the genetic selection differential for the $k$ th sex age-group expressed as a deviation from mean of the whole contemporary sex-age group and the sum is taken over all sex-age groups. Furthermore, in conventional gene flow theory, asymptotic proportions of genes are predicted from the proportional contributions of sex-age groups to the newborn offspring. With equal reproductive rates for all age groups, the asymptotic proportion follows directly from the number of parents selected from the respective sex-age group (Eqs. (11) and (12) in [2]):

$$
r_{k, \infty}=\frac{\frac{1}{2} n_{k}}{\left(N_{\operatorname{sex}(k)} L\right)}
$$

where $n_{k}$ is the number of parents selected from the $k$ th age-sex group, $N_{\mathrm{sex}(k)}$ is the total number of parents of $\operatorname{sex}(k), L$ is the generation interval calculated as the average age of parents when their offspring are born and the " $\frac{1}{2}$ " makes asymptotic proportions of genes sum to 0.5 per sex per generation. 


\section{ASYMPTOTIC PROPORTIONS OF GENES}

Recently, Bijma and Woolliams [1] have shown that, in an ongoing breeding programme, asymptotic proportions of genes predicted from equation (2) deviate systematically from true asymptotic proportions. This will be illustrated here by simulated data. Table I shows $r_{k, \infty}$ predicted from conventional gene flow theory (Eq. 2) and $r_{k, \infty}$ observed in simulated data. The population consisted of 10 one-year-old sires, 30 two-year-old sires, 20 one-year-old dams and 20 two-year old dams with 3 offspring of each sex per dam. Mass selection was performed for a trait with an initial heritability of 0.4 . Additional results are in $[1]$.

Table I. Asymptotic proportions of genes deriving from sex-age groups $\left(r_{k, \infty}\right)$, and rate of genetic gain from equation (1) $\left(\Delta G_{\text {Eqn.1 }}\right)$ using $r_{k, \infty}$ from conventional gene flow theory (i.e. Eq. (2)) or using $r_{k, \infty}$ from simulation, and genetic gain observed in simulated data $\left(\Delta G_{\text {sim }}\right)$.

\begin{tabular}{lcc}
\hline & $\begin{array}{c}\text { Conventional gene } \\
\text { flow theory }\end{array}$ & Simulation $\dagger$ \\
\hline$r_{1, \infty}$ & 0.0769 & $0.124(0.002)$ \\
$r_{2, \infty}$ & 0.2308 & $0.206(0.002)$ \\
$r_{3, \infty}$ & 0.1538 & $0.193(0.002)$ \\
$r_{4, \infty}$ & 0.1538 & $0.135(0.002)$ \\
$\Delta G_{\text {Eqn.1 }}$ & 0.313 & 0.345 \\
$\Delta G_{\text {sim }}$ & - & $0.309(0.001)$ \\
\hline
\end{tabular}

For a scheme with 10 one year old sires, 30 two year old sires, 20 one year old dams, 20 two year old dams, 3 offspring of each sex per dam, base generation heritability = 0.4 , unity phenotypic variance and mass selection. $\dagger$ Simulation results are averaged over 500 reps., s.e. are between brackets. Values are based on Bulmer's equilibrium genetic parameters [1]: $\sigma_{A \text {,eq. }}^{2}=0.34, h_{\text {eq. }}^{2}=0.36$, so that genetic selection differentials were: $0.646,0.446,0.526,0.526$. Simulation details are in [1].

In Table I, $r_{k, \infty}$ predicted from conventional gene flow theory differs substantially from simulation results. In particular, the asymptotic proportion of genes from one-year-old parents was higher than the value predicted from equation (2). The deviations of asymptotic proportions of genes from those predicted by conventional gene flow theory arise from the inheritance of selective advantage $[1,6]$, an effect ignored in conventional gene flow theory. For example, when one-year-old selected sires have a higher mean breeding value than their selected male contemporaries, offspring of those one-year-old sires will have an increased probability of being selected which increases the asymptotic proportion of genes deriving from one-year-old sires. In such a case, $r_{1, \infty}$ will be higher than the expected proportion based on the contribution of one-year-old sires to the newborn offspring. Therefore, in an ongoing selection program, equation (2) is invalid.

For the scheme in Table I, $\Delta G$ predicted from conventional gene flow theory (i.e. Eqs. (1) and (2)) was 0.313 , which is close to the 0.309 observed 
in simulated data. Thus, for the scheme in Table I, conventional gene flow theory yields an accurate prediction of genetic gain, even though asymptotic proportions of genes predicted from equation (2) deviate considerably from the true values. However, $\Delta G$ predicted from equation (1) using $r_{k, \infty}$ observed in simulated data differed from simulated $\Delta G$ (0.345 vs. 0.309$)$, indicating that using true asymptotic proportions in equation (1) does not yield a valid prediction of genetic gain.

\section{WHY CONVENTIONAL GENE FLOW THEORY GIVES A VALID PREDICTION OF $\Delta G$}

Although equation (2) is not generally valid, conventional gene flow theory yields a valid prediction of $\Delta G$. This follows from substituting equation (2) into

equation (1), which gives: $\Delta G=\sum_{k} r_{k, \infty} S_{k}=\frac{1}{2 L} \sum_{k} n_{k} S_{k} / N_{\operatorname{sex}(k)}=\bar{S} / L$. This equation is identical to the well-known result of Rendel and Robertson [5] and yields a valid prediction of the rate of genetic gain as shown unmistakably by James [4]. Therefore, equation (1) is valid only when $r_{k, \infty}$ is calculated from equation (2) even though this means that $r_{k, \infty}$ differs from the true asymptotic proportion of genes.

It can be understood intuitively why $r_{\infty, k}$ should refer to the contribution of sex-age groups to newborn offspring the next cohort in equation (1). As indicated above, the difference between asymptotic proportions predicted from conventional gene flow theory and true asymptotic proportions is due to inheritance of selective advantage which changes the proportions in subsequent cycles of selection. Though we are concerned with the asymptotic proportion of genes from specific sex-age groups, part of this proportion arises due to subsequent cycles of selection and should therefore not be attributed to genetic gain originating from a single cycle of selection. Because selection in subsequent generations favors descendents of parents with an above average breeding value, the use of true asymptotic proportions in equation (1) results in an overprediction of the rate of genetic gain.

Hopkins and James [3] studied rates of genetic gain based on contributions of parental age groups to selected offspring in the next cohort. However, true asymptotic proportions of genes are not only affected by selection among the offspring, but also by subsequent rounds of selection $[1,6]$. Therefore, asymptotic proportions of genes that can be calculated using methods in [3] will deviate systematically from true asymptotic proportions. The predicted $\Delta G$ of [3] however is valid, as shown by James [4].

\section{ANOTHER APPROACH}

By decomposing breeding values into Mendelian sampling terms, Woolliams et al., [6] have shown that the annual rate of genetic gain is equal to the product of the asymptotic proportion of genes deriving from an individual and its Mendelian sampling term, summed over all parents per year:

$$
\Delta G=\sum r_{i, \infty} a_{i}
$$


where $r_{i, \infty}$ is the asymptotic proportion of genes deriving from individual $i$ (i.e. its long term genetic contribution), $a_{i}$ is the Mendelian sampling contribution to the genotype of individual $i$ and the sum is taken over all the parents in a year. Note that equation (3) is expressed on an individual level, whereas equation (1) is expressed on a sex-age class level. In equation (3), genetic gain is attributed to the cohort in which the newly arising variation (i.e. the Mendelian sampling term) is generated. The product of the long term genetic contribution and the Mendelian sampling term quantifies the impact of an individual on the population mean in the long term. Contrary to equation (1), genetic gain originating from a specific individual or group accumulates over generations in equation (3). The convergence of genetic contributions to their equilibrium values takes several cycles of selection. During the first cycles the summed product of genetic contributions and Mendelian sampling terms is lower than $\Delta G$, but selection favors contributions that go together with above average Mendelian sampling terms and the product increases until genetic contributions stabilize and $\sum r_{i, \infty} a_{i}=\Delta G$. Equation (3) therefore considers the gain arising from a single cohort over all subsequent cycles of selection, whereas Rendel and Robertson's equation considers the gain from selection in a single cycle arising from all previous cohorts. Both are valid, whereas equation (1), in considering gain from all previous cohorts over all subsequent cycles of selection results in double counting. Using a modified gene flow procedure, Woolliams et al. [6] and Bijma and Woolliams [1] show how asymptotic proportions of genes can be predicted accurately, either on an individual or on a group level.

\section{CONCLUSION}

This note has shown that rate of genetic gain differs from the summed product of asymptotic proportions of genes and selection differentials. The connection between asymptotic proportions of genes and rate of genetic gain that is embodied in conventional gene flow theory is therefore invalid, even though genetic gain may be correctly predicted from it. Thus, rate of genetic gain may be expressed in two manners. First, from conventional gene flow theory: $\Delta G=\Sigma r_{k, 0} S_{k}$, in which case $r_{k, 0}$ denotes the proportional contribution of the $k$ th sex-age group to the newborn offspring in the next cohort, as given by equation (2). Second, $\Delta G=\sum r_{i, \infty} a_{i}$ where $r_{i, \infty}$ is the true individual asymptotic contribution. Both expressions are valid and give similar results [1]. The first expression is based on contributions to the next generation and is valid since it is identical to Rendel and Robertson's result, whereas the second is truly based on asymptotic proportions of genes. Furthermore, conventional gene flow theory can still be used to calculate discounted returns from a single cycle of selection, since differences between $r_{k, 0}$ and true asymptotic proportions originate from subsequent cycles of selection and should therefore not be attributed to a single cycle of selection. 


\section{ACKNOWLEDGEMENTS}

The research was financially supported by the Netherlands Technology Foundation (STW) and was coordinated by the Earth and Life Sciences Foundation (ALW). JAW gratefully acknowledges the Ministry of Agriculture, Fisheries and Food for financial support.

\section{REFERENCES}

[1] Bijma P., Woolliams J.A., Prediction of genetic contributions and generation intervals in populations with overlapping generations under selection, Genetics 151 (1998) 1197-1210.

[2] Hill W.G., Prediction and evaluation of response to selection with overlapping generations, Anim. Prod. 18 (1974) 117-139.

[3] Hopkins I.R., James J.W., Genetic responses in the early years of selection programmes using genetic differences between generations, Anim. Prod. 28 (1979) 65-77.

[4] James J.W., A note on selection differentials and generation length when generations overlap, Anim. Prod. 24 (1977) 109-112.

[5] Rendel J.M., Robertson A., Estimation of genetic gain in milk yield by selection in a closed herd of dairy cattle, J. Genet. 50 (1950) 1-8.

[6] Woolliams J.A., Bijma P., Villanueva B., Expected genetic contributions and their impact on gene flow and genetic gain, Genetics 153 (1999) 1009-1020. 